\title{
Rapid Bioprosthetic Mitral Valve Failure after Temporary Left Ventricular Assist Device Support
}

\author{
Heidi B. Schubmehl, MD, Muhamed Saric, MD, Alan F. Vainrib, MD, Mathew L. Williams, MD, \\ Alex Reyentovich, MD, and Leora B. Balsam, MD
}

Department of Cardiothoracic Surgery and Division of Cardiology, NYU-Langone Medical Center, New York, New York, USA

\section{ABSTRACT}

The technique of mechanical circulatory support or the site of cannulation may affect transvalvular flow. We describe early failure of a mitral bioprosthesis in a patient with temporary left ventricular assist device support, likely from thrombosis. Salvage with transcatheter mitral valve replacement was attempted. Temporary mechanical circulatory support strategies that maximize flow across a new bioprosthesis should be sought to avoid early valvular thrombosis.

\section{INTRODUCTION}

Mechanical circulatory support, including ventricular assist devices and extracorporeal membrane oxygenation (ECMO), are important adjuncts for salvage of patients with post-cardiotomy shock. Transmitral flow is reduced when left ventricular assist device (LVAD) inflow cannulation is left atrial or ECMO support is chosen. We describe a case of accelerated bioprosthetic mitral valve failure that developed following temporary LVAD support using a left atrial inflow cannulation strategy. The valvular dysfunction was not detected until transition to durable LVAD support with left ventricular inflow cannulation, at which time the patient rapidly developed cardiogenic shock.

\section{CASE REPORT}

A 52-year-old man with non-ischemic cardiomyopathy, atrial flutter, and prior bioprosthetic aortic and mitral valve replacement two years prior presented with cardiogenic shock, acute kidney injury, liver dysfunction, and thrombocytopenia. Transthoracic echocardiogram revealed a thrombosed prosthetic mitral valve (gradient $19 \mathrm{mmHg}$ ), left ventricular ejection fraction of $5 \%$, and left ventricular end-diastolic dimension of 8.2 centimeters. He was treated with inotropes but remained critically ill with end-organ dysfunction. Considering the severity of his cardiomyopathy, end-organ damage and need for reoperative surgery, staged operations of redo mitral valve replacement with temporary

Received Fune 25, 2017; accepted August 3, 2017.

Leora Balsam, MD, Division of Cardiac Surgery, UMass Memorial Medical Center, 55 Lake Avenue North, Worcester, MA 01655 (email: leora.balsam@ umassmemorial.org). left heart mechanical support followed by delayed conversion to a Heartmate II LVAD (Abbott, Pleasanton, CA, USA) were planned. He underwent prosthetic mitral valve replacement with a $29 \mathrm{~mm}$ Epic bioprosthesis (St. Jude Medical, Saint Paul, MN, USA) and Centrimag LVAD (Abbott, Pleasanton, CA) implantation with left atrial inflow and ascending aortic outflow cannulation (CPB 229 minutes, cross-clamp 127 minutes). At a speed of $3100 \mathrm{rpm}$, centrimag flow was over $4 \mathrm{~L} / \mathrm{min}$, and he had steady improvement of endorgan function. Anticoagulation with therapeutic heparin was initiated on postoperative day 1. On postoperative day 5, he underwent Centrimag explantation and Heartmate II implantation (CPB 136 minutes). Within hours, his lactate rose from 6 to $12 \mathrm{mmol} / \mathrm{L}$ and he developed severe end-organ dysfunction. LVAD speed was $8200 \mathrm{rpm}$ with flow of $3.5 \mathrm{~L} /$ min, pulsatility index of 2-3, and power of 4 watts. Central venous pressure was $15 \mathrm{mmHg}$ and pulmonary artery pressure was $60 / 33$ (mean 40) mmHg. He was taken back to the

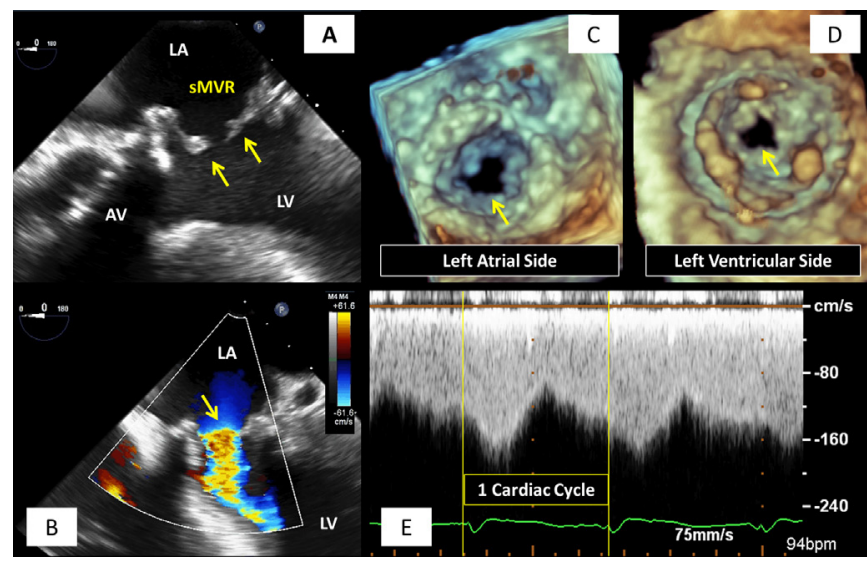

Figure 1. Acute bioprosthetic mitral valve stenosis. A, Mitral bioprosthetic leaflets are thickened and immobile (arrow). B, High velocity aliased flow (arrow) across the mitral bioprosthesis is noted on color Doppler imaging, consistent with mitral stenosis. C and D, 3-dimensional TEE images demonstrate marked decrease in mitral orifice (arrow) with no change in orifice size between systole and diastole (frozen leaflets). E, Continuous wave spectral Doppler tracing across the mitral bioprosthesis demonstrates high velocity transvalvular flow consistent with mitral stenosis. There is continuous antegrade flow across the prosthesis due to functioning continuous flow LVAD. AV indicates aortic valve; LA, left atrium; LAA, left atrial appendage; LV, left ventricle; sMVR, surgical mitral valve replacement. 


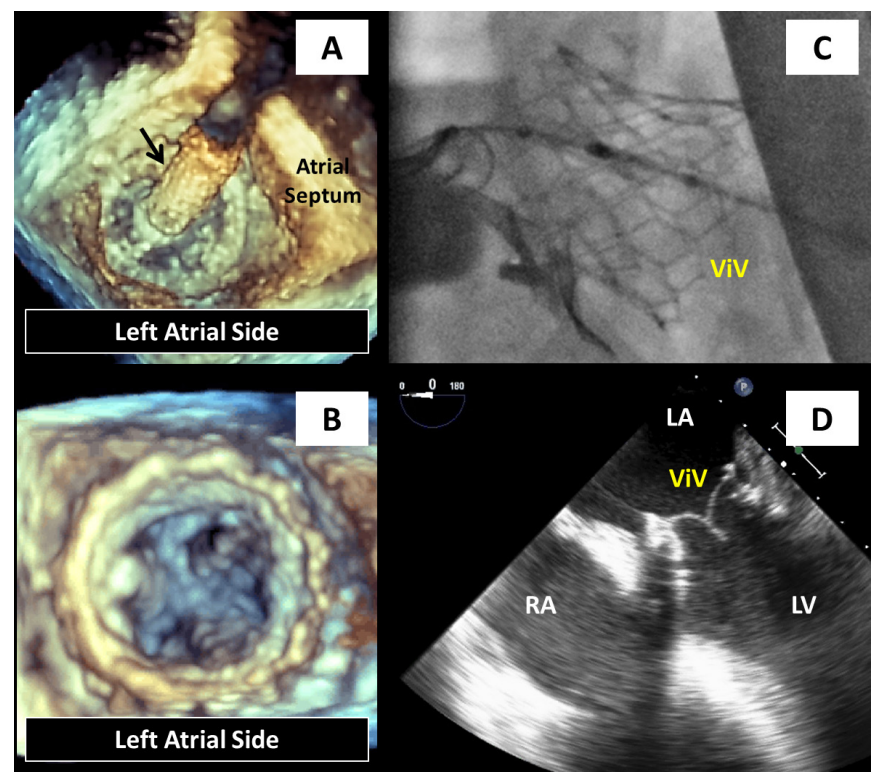

Figure 2. Valve-in-valve procedure. A, 3D-TEE of left atrial aspect of surgical mitral bioprosthesis demonstrates passage of a folded Sapien 3 transcatheter valve (arrow) through a right-sided pulmonary vein prior to its deployment. B, 3D-TEE demonstrates left atrial aspect of fully deployed valve-in-valve. C, Valve-in-valve deployment visualized by fluoroscopy. D, 2D-TEE demonstrates fully deployed mitral valve-in-valve. LA indicates left atrium; LV, left ventricle; RA, right atrium; ViV, valvein-valve.

operating room for reexploration. Transesophageal echocardiography revealed thickened, immobile bioprosthetic mitral valve leaflets with severe valvular stenosis (estimated valve area $0.4-0.5 \mathrm{~cm}^{2}$ ), presumably secondary to acute thrombosis (Figure 1). Right ventricular function was moderately depressed and the left ventricle was underfilled. Transcatheter valve-in-valve mitral replacement through the right pulmonary vein was performed with a $29 \mathrm{~mm}$ Sapien 3 valve (Edwards Lifesciences, Irvine, CA, USA) in a salvage attempt (Figure 2). Although the transvalvular gradient was relieved, implantation was complicated by air-entrainment, resulting in right ventricular collapse from intracoronary air. A temporary right ventricular assist device was placed. Despite these measures, the patient's multi-organ failure progressed and he expired.

\section{DISCUSSION}

Transvalvular flow across a newly implanted bioprosthesis may be affected by severity of native cardiac dysfunction and mechanical circulatory support strategies, including mode of support (VAD versus ECMO) and site of cannulation. We selected the left atrium for inflow cannulation for the temporary LVAD because it was readily accessible, and exposure of the left ventricular apex would have added considerable cardiopulmonary bypass time. Although the patient was maintained on inotropes to allow for native left ventricular ejection, it is likely that transmitral flow remained severely compromised. Time to development of the bioprosthetic mitral valve failure is unclear, as it did not become clinically manifest until after HeartMate II placement. With its left ventricular inflow cannula, the patient became dependent on transmitral flow for device filling. This case highlights the role of systematic transesophageal echocardiography at the time of durable VAD implantation as well as the need to maintain tranvalvular flow across a newly implanted bioprosthetic valve.

Whether the new bioprosthesis thrombosed or the valve leaflets fused is unknown, as post-mortem examination was not performed. Bioprosthetic valvular thrombosis and fusion have been reported by other authors in the setting of mechanical circulatory support [Hagley 1995; Kagiyama 2014; Mufti 2013]. Kagiyama et al [Kagiyama 2014] described two patients with prosthetic mitral valve thrombosis after peripheral venoarterial ECMO for post-cardiotomy shock. Mufti et al [Mufti 2013] found mitral valve fusion post-mortem in a patient with biventricular assist devices for post-cardiotomy failure after mitral valve replacement. In that case, LVAD inflow cannulation was through the right superior pulmonary vein. The valve pathology was not recognized until device explantation 20 days later, at which time the patient developed low cardiac output syndrome and death.

In conclusion, when planning temporary ventricular support for patients with new bioprostheses, strategies that maximize transvalvular flow may reduce the likelihood of early valve failure. Although more attention has been paid to the risk of thrombosis of mechanical valves in this setting, bioprostheses are also at risk.

\section{REFERENCES}

Hagley MT, Lopez-Candales A, Phillips KJ, et al. 1995. Thrombosis of mitral valve bioprostheses in patients requiring circulatory assistance. Ann Thorac Surg 60:1814-16.

Kagiyama N, Okura H, Nezuo S, et al. 2014. Two cases of acute bioprosthetic mitral valve thrombosis immediately after mitral valve replacement. Circulation 129:e328-30.

Mufti HN, Elghobary T, Murray SK, et al. 2013. Mitral valve and shortterm ventricular assist devices: potential mechanical complications. Thorac Cardiovasc Surg Rep 2:9-12. 